\title{
CORRIGENDUM
}

\section{Meta-analysis of five genome-wide linkage studies for body mass index reveals significant evidence for linkage to chromosome $8 p$}

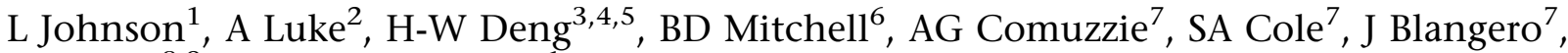 \\ M Perola $^{8,9}$ and M Dawn Teare ${ }^{1}$
}

International Journal of Obesity (2005) 29, 878. doi:10.1038/sj.ijo.0802966

Correction to: International Journal of Obesity (2005) 29, 413-419. doi: 10.1038/sj.ijo.0802817

Due to an author error, the authors and affiliation list for the above article were published incorrectly. The correct list of authors and affiliations are reproduced below.

L Johnson $^{1}$, A Luke ${ }^{2}$, A Adeyemo ${ }^{3,4}$, H-W Deng ${ }^{5,6,7,8}$, BD Mitchell $^{9}$, AG Comuzzie $^{10}$, SA Cole ${ }^{10}$, J Blangero ${ }^{10}, \mathrm{M}$ Perola $^{11,12}$ and MD Teare ${ }^{1 *}$

${ }^{1}$ Mathematical Modelling and Genetic Epidemiology, Division of Genomic Medicine, University of Sheffield, UK; ${ }^{2}$ Department of Preventive Medicine and Epidemiology, Loyola University Stritch School of Medicine, Maywood, IL, USA; ${ }^{3}$ College of Medicine, University of Ibadan, Ibadan, Nigeria; ${ }^{4}$ Genetic Epidemiology Unit, National Human Genome Center, Howard University, Washington, DC, USA; ${ }^{5}$ Osteoporosis Research Centre, Creighton University, Omaha, NE, USA; ${ }^{6}$ Department of Biomedical Sciences,
Creighton University, Omaha, NE, USA; ${ }^{7}$ Molecular and Statistical Genetics Lab, Hunan Normal University, ChangSha, China; ${ }^{8}$ The Key Laboratory of Biomedical Information Engineering of Ministry of Education and Institute of Molecular Genetics, School of Life Science and Technology Xi'an Jiaotong University, China; ${ }^{9}$ Department of Medicine, University of Maryland School of Medicine, Baltimore, MD, USA; ${ }^{10}$ Department of Genetics, Southwest Foundation for Biomedical Research, San Antonio, USA; ${ }^{11}$ Department of Molecular Medicine, National Public Health Institute University of Helsinki, Helsinki, Finland; and ${ }^{12}$ Department of Human Genetics, Gonda Neuroscience and Genetics Research Centre, University of California-Los Angeles, USA

*Correspondence: Dr MD Teare, Mathematical Modelling and Genetic Epidemiology, Division of Genomic Medicine, University of Sheffield Medical School, Beech Hill Road, Sheffield S10 2RX, UK.

E-mail: m.d.teare@sheffield.ac.uk 